COLORECTAL CANCER

\title{
Targeting BRAF mutations equally?
}

Patients with colorectal cancer (CRC) who harbour BRAF mutations have a very poor prognosis. Although the BRAF inhibitor vemurafenib has shown remarkable activity in patients with melanoma, this activity has not been well characterized in patients with CRC. On the basis of the recommended phase II dose of vemurafenib in patients with melanoma, an expansion cohort was conducted in 21 patients with $B R A F^{{ }^{6} 600 E}$-mutated metastatic CRC. Vemurafenib monotherapy did not show marked clinical activity in these patients: the median progression-free survival was 2.1 months, and the median overall survival 7.7 months.

Total EGFR expression was assessed in the expansion cohort because preclinical research had shown that activation of PI3K signalling might be associated with resistance to BRAF inhibition, via EGFR feedback activation. In the expansion cohort, no correlation was observed between total EGFR expression in the tumour and clinical activity. However, concurrent KRAS and NRAS mutations were detected at a low frequency, indicating possible mechanisms of acquired resistance to vemurafenib. PTEN loss and PIK3CA mutation, however, did not correlate with resistance.

Preclinical data published in Nature showed that inhibition of $B R A F^{\mathrm{V} 600 \mathrm{E}}$ in colon cancers caused rapid feedback activation of EGFR, which drives continued tumour proliferation. Melanoma cells express low levels of EGFR and are not subject to this feedback activation. Thus, insufficient MAPK-pathway inhibition by vemurafenib might be a reason for the lack of clinical activity in CRC, providing a rationale for using BRAF and EGFR combination therapy.

Scott Kopetz, lead author of the study, comments: "these latest phase II results in patients with CRC have implications for conducting oncogene-defined basket clinical trials."

Lisa Hutchinson

ORIGINAL ARTICLE Kopetz, S. et al. Phase ॥ pilot study of vemurafenib in patients with metastatic BRAF-mutated colorectal cancer. J. Clin. Oncol. doi:10.1200/JCO.2015.63.2497 FURTHER READING Prahallad, A. et al.

Unresponsiveness of colon cancer to BRAF(V600E) inhibition through feedback activation of EGFR.

Nature 483, 100-103 (2012) 